\title{
PERCEPÇÕES DA EQUIPE DE SAÚDE E DE FAMILIARES SOBRE A CONSULTA DE PUERICULTURA*
}

Tatiana da Silva Melo Malaquias ${ }^{1}$, Vanessa Antoniassi Denardi Baldissera², leda Harumi Higarashi ${ }^{3}$

'Enfermeira. Mestre em Enfermagem. Professora da Universidade Estadual do Centro-Oeste. Guarapuava-PR-Brasil.

${ }^{2}$ Enfermeira. Doutora em Enfermagem. Professora da Universidade Estadual de Maringá. Maringá-PR-Brasil.

${ }^{3}$ Enfermeira. Doutora em Educação. Professora da Universidade Estadual de Maringá. Maringá-PR-Brasil.

RESUMO: Estudo qualitativo descritivo com o objetivo de conhecer a dinâmica da consulta de puericultura de equipes da Estratégia Saúde da Família de um Núcleo Integrado de Saúde e seus reflexos nas concepções da equipe de saúde e de familiares de crianças inscritas no Programa de Puericultura. A coleta de dados ocorreu entre junho de 2012 e fevereiro de 2013, mediante entrevista semiestruturada e observação não participante e submetidos à análise de conteúdo. Foram sujeitos, 19 profissionais de saúde e 13 familiares de crianças. Os resultados apontaram fragilidades na operacionalização das consultas e que o saber incipiente dos profissionais acerca do tema, contribui para uma prática fragmentada, pautada no curativismo, acarretando manutenção de concepções errôneas entre os usuários do sistema. Sinaliza-se a necessidade de conscientização dos profissionais, com vistas à construção de um novo paradigma nesta realidade, que valorize a consulta de puericultura, enquanto ferramenta imprescindível à promoção da saúde infantil. DESCRITORES: Saúde da criança; Desenvolvimento infantil; Pessoal de saúde; Família; Programa saúde da família.

\section{PERCEPTIONS OF THE HEALTH TEAM AND FAMILY MEMBERS REGARDING THE CHILDCARE CONSULTATION}

ABSTRACT: This qualitative descriptive study aimed to investigate the dynamic of the childcare consultation of Family Health Strategy teams of an Integrated Health Nucleus and reflections of this in the conceptions of the health team and the family members of children registered on the Childcare Program. Data collection took place in June 2012 - February 2013, through semistructured interviews and non-participant observation, and were subjected to content analysis. The subjects were 19 health professionals and 13 family members of children. The results indicated weaknesses in the operationalization of the consultations and that the professionals' incipient knowledge regarding the issue contributes to fragmented practice based in curativism, resulting in the maintaining of erroneous conceptions among the system's users. The need is indicated to raise professionals' awareness, with a view to the construction of a new paradigm in this context, valuing the childcare consultation as an essential tool for the promotion of child health. DESCRIPTORS: Child health; Child development; Health personnel; Family; Family health program.

\section{PERCEPCIONES DEL EQUIPO DE SALUD Y DE FAMILIARES ACERCA DE LA CONSULTA DE PUERICULTURA}

RESUMEN: Estudo cualitativo descriptivo cuyo objetivo fue conocer la dinámica de la consulta de puericultura de equipos de Estrategia Salud de la Familia de un Nucleo Integrado de Salud y sus reflejos en las concepciones del equipo de salud y de familiares de niños del Programa de Puericultura. Los datos fueron obtenidos entre junio de 2012 y febrero de 2013, por medio de entrevista semiestructurada y observación no participante y sometidos al análisis de contenido. Participaron 19 profesionales de salud y 13 familiares de niños. Los resultados apuntaron fragilidades en la operacionalización de las consultas y que el saber incipiente de los profesionales acerca del tema contribuye para una práctica fragmentada, pautada en el "curativismo", traendo manutención de concepciones erróneas entre los usuarios del sistema. Se apunta la necesidad de conscientización de los profesionales para que sea posible contruir un nuevo paradigma en esta realidad, lo cual valore la consulta de puericultura, en cuanto herramienta imprescindible a la promoción de la salud infantil. DESCRIPTORES: Salud del niño; Desarrollo infantil; Personal de salud; Familia; Programa Salud de la Familia.

*Artigo extraído da Dissertação intitulada: "Percepção da equipe de saúde e dos familiares da criança sobre a consulta de puericultura". Universidade Estadual de Maringá, 2013. 


\section{INTRODUÇÃO}

Na atualidade, a Atenção à Saúde da Criança no Brasil é considerada um eixo prioritário do Sistema de Saúde, sendo vinculada ao Ministério da Saúde (MS) pela Área Técnica de Saúde da Criança e Aleitamento Materno (ATSCAM). Os modelos de atenção propostos pela ATSCAM são voltados à promoção de saúde, vigilância, prevenção e assistência em "linhas de cuidado", visando à atenção integral da saúde da criança, dentre elas: Atenção à Saúde do Recém-Nascido; Promoção, Proteção e Apoio ao Aleitamento Materno; Prevenção de Violências e Promoção da Cultura da Paz; Incentivo e Acompanhamento do Crescimento e Desenvolvimento ${ }^{(1)}$.

O Ministério da Saúde propõe a Agenda de Compromissos para a Saúde Integral da Criança e Redução da Mortalidade Infantil para a orientação da ação de todos os profissionais que lidam com a criança. O objetivo deste documento é garantir que a criança possa se beneficiar de um cuidado integral e multiprofissional, que dê conta de compreender todas as suas necessidades e direitos como indivíduo. Como cuidado integral entende-se a responsabilidade de disponibilizar a atenção necessária em todos os níveis: da promoção à saúde no nível mais complexo de assistência, do locus próprio da atenção à saúde aos demais setores que têm interface estreita e fundamental com a saúde (moradia, água tratada, educação, etc) ${ }^{(2)}$.

Desta forma, o atendimento integral à saúde da criança mostra-se como um importante marco na assistência infantil e no reconhecimento dos direitos da criança, possibilitando o crescimento e desenvolvimento saudável com todo seu potencial $^{(3)}$.

O acompanhamento do crescimento e desenvolvimento infantil no contexto da Estratégia Saúde da Família (ESF) tem no Programa de Puericultura (PP) o seu eixo norteador, razão pela qual as atividades que integram o mesmo devem ser desenvolvidas por um grupo de profissionais comprometidos com o cuidado infantil.

Neste cenário, a consulta de puericultura tem como objetivo a promoção da saúde infantil, aliada à prevenção de doenças e agravos futuros. Para que esta ocorra de maneira satisfatória, o profissional responsável pela consulta deve conhecer o Programa de Puericultura preconizado pelo MS e os objetivos a que se propõe, além de compreender a criança no ambiente familiar e social e as relações culturais e econômicas nas quais está inserida ${ }^{(4)}$.

$\mathrm{Na}$ "Primeira Semana Saúde Integral", que compreende as ações que são desenvolvidas na primeira semana de vida do neonato e objetivam o fortalecimento do vínculo mãe-filho e a redução da mortalidade materna e infantil, todo recémnascido deve ser acolhido na Unidade Básica de Saúde para checagem dos cuidados tanto para a mãe quanto para a criança, ressaltando-se a importância dessa abordagem nos primeiros dias de vida e primeiro mês, quando ocorre a maioria dos problemas que levam ao desmame precoce ${ }^{(2)}$.

Neste sentido, o início das consultas de puericultura deve ser realizado até 15 dias de vida do recém-nascido. A faixa etária para os atendimentos compreende desde o nascimento até os dez anos. Os atendimentos podem ser realizados pelo médico ou enfermeiro e os retornos devem ser agendados conforme a necessidade de cada caso (diário, semanal, quinzenal ou mensal) ${ }^{(5)}$.

Ademais, salienta-se que a assistência à saúde da criança deve privilegiar os sentimentos e valores das famílias, de modo que todos os sujeitos envolvidos no processo de cuidado possam tomar decisões conjuntas e necessárias à promoção e recuperação da vida saudável. Nessa perspectiva, a relação entre profissionais e usuários possibilita a busca da autonomia e a capacidade de mudanças na sociedade a que pertencem $^{(6)}$.

Assim, a empatia, o atendimento humanizado e a formação de um vínculo afetivo entre a equipe de saúde e as famílias, se destacam enquanto ferramentas que favorecerão a adesão ao PP, possibilitando desta forma, o adequado seguimento das crianças. Não obstante a compreensão desta cadeia de ações seja simples, verifica-se nas realidades assistenciais a existência de lacunas no que tange à sua efetivação. Desta constatação, surgiram as inquietações que nos levaram à proposição de um estudo que buscasse conhecer como a consulta de puericultura se encontra inserida no "fazer" cotidiano dos profissionais de saúde de ESF e qual a compreensão que os familiares das crianças atendidas pela estratégia saúde da família têm sobre este atendimento. 
O objetivo deste estudo foi conhecer a dinâmica da consulta de puericultura das equipes da Estratégia Saúde da Família de um Núcleo Integrado de Saúde e seus reflexos nas concepções da equipe de saúde e dos familiares das crianças inscritas no Programa de Puericultura.

\section{MÉTODO}

Estudo de abordagem qualitativa descritiva, realizado no município de Maringá, estado do Paraná, junto às equipes da ESF atuantes em um Núcleo Integrado de Saúde, tendo como participantes profissionais das equipes de saúde, vinculados ao Programa de Puericultura e familiares das crianças inscritas no referido programa.

A rede básica de saúde de Maringá conta na atualidade com 34 Unidades Básicas de Saúde (UBS), organizadas em 5 regionais - os Núcleos Integrados de Saúde (NIS), onde atuam as equipes da Estratégia Saúde da Família. Em 2013, a cobertura era de $61,97 \%$ da população, com 66 equipes da $\operatorname{ESF}^{(7-8)}$.

O locus selecionado para a realização do presente estudo foi o Núcleo Integrado de Saúde (NIS) Pinheiros, eleito em função de constituirse em campo de práticas de ensino, pesquisa e extensão dos cursos da área de saúde da Universidade Estadual de Maringá. No período de realização da pesquisa havia sete equipes da ESF atuantes no NIS, denominadas equipe 02, equipe 03, equipe 04, equipe 05, equipe 06, equipe 07 e equipe 10. Cada equipe da ESF contava com um enfermeiro, um médico, técnico ou auxiliar de enfermagem e agentes comunitários de saúde.

A atenção à saúde da criança nas equipes da ESF é efetivada com base no Programa Nacional de Imunização e no Programa de Puericultura, sendo que o médico e o enfermeiro são os profissionais responsáveis pelas consultas, recebendo apoio dos auxiliares/técnicos em enfermagem para a verificação dos dados antropométricos e agentes comunitários de saúde nas visitas domiciliares. As atividades que compõe a puericultura na referida realidade, incluíam as consultas agendadas e as visitas domiciliares.

As crianças são acompanhadas de 0 a 10 anos de idade e as consultas são agendadas tendo como diretriz o padrão estabelecido pelo Ministério da
Saúde, no Caderno de Atenção Básica: Saúde da Criança - Crescimento e Desenvolvimento ${ }^{(5)}$.

A coleta de dados ocorreu no período de junho de 2012 a fevereiro de 2013, por meio de entrevistas utilizando um roteiro semi-estruturado e pela observação não participante das consultas de puericultura pelas pesquisadoras. Para a observação foi utilizado um roteiro, em formato de checklist, constando os dados sobre as atividades e procedimentos realizados na consulta, recursos e materiais utilizados, registros e sistemas de informação/comunicação, relacionamento com a criança e seus familiares, agendamento das consultas, controles de informações e preenchimento do cartão da criança.

Os critérios de inclusão para os familiares foram: ter um filho $^{(a)} /$ neto $^{(a)}$ inscrito no PP e estar em acompanhamento há pelo menos 6 meses. Para os profissionais de saúde foram: ser profissional da equipe de saúde da Estratégia Saúde da Família (médico, enfermeiro, técnico ou auxiliar em enfermagem e agente comunitário de saúde) há pelo menos um ano e atuar em atividades relacionadas à consulta de puericultura.

Nas entrevistas com os participantes, foram utilizados dois modelos de roteiro semiestruturado: um para os profissionais de saúde e outro para os familiares da criança, constituídos de duas partes: uma voltada à caracterização dos sujeitos de pesquisa, e outra, contendo a questão norteadora pertinente à abordagem da temática central do estudo e questões de amparo. A questão norteadora para os profissionais foi: Qual a sua percepção sobre a consulta de puericultura no contexto da atenção à saúde da criança? Para os familiares: Qual a sua percepção sobre a consulta de puericultura para a saúde de seu filho/ neto? Tal participação se deu mediante instrução prévia e assinatura do Termo de Consentimento Livre e Esclarecido. As entrevistas foram gravadas em áudio e, posteriormente, transcritas na íntegra para os procedimentos de análise. Os dados foram coletados até atingir os objetivos da pesquisa.

Para preservar a identidade dos entrevistados, os familiares foram identificados com o nome de pássaros e os profissionais de saúde com o nome de flores. Os dados extraídos das observações foram identificados como "OE' (observação da equipe) seguido do número atribuído à equipe $(2,3,4,5,6,7,10)$. 
O processo de interpretação dos dados se deu por meio da análise categorial temática ${ }^{(9)}$, na qual o material foi primeiramente organizado, para posterior transcrição. Foram implementadas diversas leituras para apreender as unidades de registro significativas, com vistas a buscar atender os objetivos da pesquisa. Na sequência, ocorreu o aprofundamento analítico em que as unidades foram agrupadas por semelhanças e divergências, formando temas que conduziram às inferências, estabelecendo assim, a categoria temática do estudo discutida com embasamento teórico e reflexivo: A dinâmica das consultas de puericultura nas ESF.

O protocolo de estudo (CAAE 05191612.2.0000.0104) foi aprovado pelo Comitê Permanente de Ética em Pesquisas Envolvendo Seres Humanos da Universidade Estadual de Maringá (COPEP), conforme os pareceres $n^{\circ}$ $63568 / 2012$ e 147.735/2012 e seguiu todos os preceitos estabelecidos pela Resolução 466/2012 do $\mathrm{CNS} / \mathrm{MS}$.

\section{RESULTADOS}

\section{Caracterização dos sujeitos da pesquisa}

A pesquisa teve como participantes dezenove familiares de crianças inscritas no Programa de Puericultura, sendo dezesseis mães, duas avós e um pai, além de treze profissionais de saúde, dos quais sete eram agentes comunitários de saúde (ACS), três médicos, duas enfermeiras e uma auxiliar de enfermagem.

\section{A dinâmica das consultas de puericultura nas ESF}

Durante as observações das consultas de puericultura, foi possível verificar configurações variadas no que tange ao protagonismo de sua implementação: nas equipes três e sete a puericultura era desenvolvida pelo médico da família; na equipe quatro era a enfermeira quem respondia integralmente pela consulta; nas equipes seis e dez era a pediatra que atendia as crianças; na equipe dois a enfermeira e o médico desenvolviam a consulta; a na equipe cinco a consulta de puericultura não estava sendo realizada em virtude da falta do profissional médico (OE 2, 3, 4, 5, 6, 7 e 10).
No desenvolvimento da pesquisa, verificouse que outras duas equipes, privadas do médico da família, deixaram de realizar a puericultura. Nestes dois casos, as crianças inscritas passaram a ser encaminhadas à médica pediatra do NIS (OE 6 e 10).

Em uma das equipes da ESF, na qual a consulta de puericultura era realizada pelo médico da família (clínico geral) constatouse que a abordagem da criança se dava de forma superficial, sem levar em consideração as diretrizes estabelecidas pelo Ministério da Saúde. Nestes atendimentos, as medidas antropométricas das crianças eram verificadas previamente pelas auxiliares de enfermagem e encaminhadas ao médico pelo sistema informatizado. Não foi realizada a conferência dos dados e marcação no Cartão da Criança pelo médico, tampouco foi informado ao familiar como estava a evolução do acompanhamento do crescimento e desenvolvimento da criança (OE 3).

A maioria das consultas de puericultura realizada pelas equipes baseava-se, prioritariamente, no modelo curativista, apoiando-se na dimensão biológica do adoecer da criança. Percebeu-se ainda que alguns dos profissionais e familiares participantes da pesquisa não possuíam uma noção mais aprofundada acerca dos princípios que balizam a consulta de puericultura (OE 2, 3, 6, 7 e 10):

O médico pergunta se está tudo bem, se precisa de alguma coisa e escuta o coração da minha filha. (Maritaca - mãe)

Eu precisei de orientação com esse médico e ele não soube me dizer (ele não é pediatra, né!?) Ele precisa dar mais atenção, olhar um pouco mais para a criança. (Gaivota - mãe)

Puericultura é o acompanhamento do desenvolvimento infantil. (Margarida - Enfermeira)

Ah, puericultura? É verificar o peso, acompanhar a criança, o crescimento. Deve ser feita pelo médico. (Violeta - Agente Comunitário de Saúde)

Puericultura? Não sei o que é não. (Arara-Azul - mãe) 
Puericultura? Já ouvi falar, mas não sei o que significa. (Rouxinol - mãe)

A consulta de puericultura foi realizada pela enfermeira em apenas uma das sete equipes das ESF observadas. Notou-se que a consulta era mais organizada e seguia uma sistematização equivalente ao que é proposto pelo Ministério da Saúde. A enfermeira recebeu os familiares com carinho e empatia, verificou os dados antropométricos das crianças, executou o exame físico, realizou as anotações no Cartão da Criança e no prontuário, fez orientações consonantes às necessidades da criança e da família, marcou o retorno da consulta e incentivou o seguimento do acompanhamento. Em dois atendimentos, as crianças estavam com problemas respiratórios e por esse motivo foram encaminhadas ao médico da família, que deu prosseguimento ao atendimento (OE 4):

É muito bom o atendimento da enfermeira. Ela faz com amor, dá atenção para meu filho. Ela examina tudo: o pulmão, a cabeça, a orelhinha, a barriguinha. Enfim, se eu tenho alguma dúvida, ela sempre me orienta. (Pardal- mãe)

Eu gosto do atendimento da enfermeira, ela orienta, examina a criança e se precisar de algo mais específico, ela encaminha para o médico. Procuro vir em todas as consultas agendadas, sei que é bom para minha filha. (Faisão - mãe)

A enfermeira é bem presente. Ela procura seguir corretamente os atendimentos, pois nem todas as equipes fazem desta forma. (Hortênsia - Agente Comunitário de Saúde)

Apesar da consulta de puericultura realizada pela enfermeira se aproximar do modelo estabelecido pelo Ministério da Saúde, algumas mães demonstraram certa insegurança, valorizando a consulta médica e a medicalização na abordagem da criança:

A enfermeira atende bem, mas acho que tem que ser o médico, né!? Ele vê o que a criança tem e já resolve o problema. (Bem-Te-Vi -mãe)

Acho que a criança deveria consultar com o pediatra, né!? A enfermeira é que atende aqui, mas um pediatra seria melhor, pois já dá o remédio certo. (Maritaca - mãe)

\section{DISCUSSÃO}

O Programa de Puericultura e o Programa Nacional de Imunização se apresentam, na atualidade, como os principais instrumentos de promoção e prevenção à saúde infantil. Ficou evidenciado no estudo que apesar da consulta de puericultura estar implantada nas ESF, existem algumas lacunas para sua implementação. Não foi possível observar uma sistemática única e consistente de operacionalização da consulta, o que contribui para o delineamento de um contexto assistencial sem uniformidade de ações e com uma prática heterogênea entre as equipes.

A implementação efetiva da consulta de puericultura na ESF é considerada prioritária e de extrema importância na assistência à criança e sua família, uma vez que as ações voltadas à saúde da criança propostas pelo MS têm como objetivo a assistência integral e humanizada, valorizando o paciente e sua família em seu contexto socioeconômico e cultural, deslocando o enfoque da assistência médico-curativista ${ }^{(10)}$.

A falta de valorização destes preceitos fundamentais pelos membros das equipes conduz a uma prática eminentemente técnica, traduzida por um processo de acolhimento pouco eficaz e um atendimento mecanizado por parte dos profissionais. A observação das consultas de puericultura realizadas pelas equipes revelou a falta de diálogo, pouca ou nenhuma orientação, e a ausência de espaços para que os familiares pudessem expressar seus anseios e dúvidas em relação ao cuidado com a criança. Tal postura contribui, em última instância, para o distanciamento entre profissional e usuário dos serviços, perpetuando o paradigma de uma atenção em saúde meramente assistencialista e focada no atendimento de queixas e agravos de saúde.

A literatura revela que o profissional que pratica a consulta de puericultura deve desenvolver seu trabalho com clínica ampliada, que tem como objetivo aumentar a autonomia do usuário do serviço de saúde, desempenhando o papel de educador e orientador no contexto da saúde da criança e de seus familiares, assegurando os 
melhores cuidados e fortalecendo o vínculo dos usuários com os serviços de saúde(11).

Valorizar a escuta e o diálogo são atitudes que revelam a disposição de entender o outro em suas necessidades, demonstrando o interesse do profissional em compreender os pais em suas limitações e fortalezas no cuidado à criança. Os usuários do sistema de saúde, ao avaliarem a integralidade da atenção, não levam em consideração apenas os aspectos estruturais, mas mostram-se indignados quanto à maneira pela qual se efetivam as relações, pois o cuidado só é possível se há condições concretas para o diálogo ${ }^{(3)}$.

Os pressupostos do cuidado centrado na família são a dignidade e o respeito: os profissionais ouvem e respeitam as escolhas e perspectivas do paciente e família, incorporandoos ao planejamento da assistência infantil, a fim de efetivar o vínculo e a autonomia do cuidado ${ }^{(12)}$.

Corroborando com esta concepção, um estudo realizado em Campo Grande constatou que, durante as consultas de puericultura, as mães têm a oportunidade de questionar e aprender sobre a saúde de seu filho ${ }^{(13)}$. Quando o atendimento é permeado de atenção e carinho, possibilita um relacionamento de confiança entre os familiares das crianças e os profissionais de saúde, sendo valorizado por meio do adequado seguimento das consultas pelos pais e responsáveis.

Os achados do presente estudo evidenciaram que a maioria dos profissionais que atuam na consulta de puericultura, ainda possuía a visão curativista de atendimento ao usuário, valorizando a consulta fragmentada e centrada em uma queixa a ser analisada. Como reflexo deste tipo de atendimento, a comunidade assistida pelas ESF passou a absorver as concepções dos profissionais, dando pouca importância à consulta de puericultura.

De modo semelhante, essas concepções foram apontadas em outros estudos, como na pesquisa realizada na Paraíba, e que constatou que as ações das enfermeiras nos atendimentos às crianças fundamentavam-se na dimensão biológica e no adoecer, sem agendamento prévio e livre demanda, inviabilizando a abordagem centrada no usuário ${ }^{(14)}$. No Ceará( ${ }^{(4)}$ uma pesquisa apontou que a maioria dos profissionais de saúde da ESF priorizava o atendimento a criança doente, não realizando a consulta de puericultura. Corroborando com estes dados, mães de Sobral no Ceará relataram que não levavam seus filhos nas consultas de puericultura por considerarem que os mesmos estavam sadios e que, portanto, não necessitavam atendimento ${ }^{(15)}$.

Este modelo de saúde curativista contraria frontalmente as diretrizes da Atenção Básica, representada pela ESF, e que reconhece no Programa de Puericultura, uma ferramenta oportuna para o acompanhamento do crescimento e desenvolvimento infantil, voltado aos aspectos de promoção e prevenção da saúde, proporcionando o atendimento humanizado e integral à criança, desmitificando o enfoque centrado apenas na doença ${ }^{(16)}$.

Ao assumir a mudança deste paradigma assistencial, por meio da substituição do modelo curativo pelo preventivo, com vistas à promoção da saúde, a ESF poderá se tornar um instrumento fundamental para o empoderamento da população, conduzindo-a a autonomia do cuidado $^{(17)}$.

Os dados deste estudo revelaram ainda que há profissionais comprometidos com a saúde infantil e que se preocupam em assistir à criança e sua família integralmente. Isso foi observado em apenas uma das equipes, na qual a puericultura era realizada pela enfermeira. Assim, e não obstante tratar-se de iniciativa elogiável e reconhecida pela população atendida, esta situação suscita outra problemática, que é a da falta de uma diretriz de atuação entre as equipes e a escassez de políticas de formação continuada ou de educação permanente em saúde, com vistas à qualificação da atenção.

Deste modo, pode-se inferir que, da concepção adequada sobre a consulta de puericultura dos profissionais, e da valorização deste corpo de conhecimentos pelos serviços, dependem a prática e efetivação das ações no cotidiano da atenção, e destas, por sua vez, dependem a avaliação pelos usuários dos serviços de saúde, e o processo de conscientização da própria população.

Há, pois, que ressaltar-se que, as ações desenvolvidas pela equipe de saúde são percebidas pelos usuários por meio das atitudes e comportamentos durante a dinâmica assistencial. A população associa o bom atendimento à questão vocacional, entendendo que os profissionais que desempenham o cuidado humanizado gostam do que fazem ${ }^{(4)}$. 
Mediante o conhecimento da realidade vivenciada e das condições socioeconômicas e culturais das famílias, o profissional de saúde poderá oferecer atendimento que abrange as reais necessidades de saúde da criança, com possibilidades de maior adesão ao tratamento e orientações, favorecendo o desenvolvimento infantil com o mínimo de agravos para que alcance uma vida adulta saudável ${ }^{(3-4)}$.

A literatura aponta que várias são as estratégias que favorecem o envolvimento da comunidade no processo de autonomia do cuidado. Podese destacar a educação em saúde como uma ferramenta facilitadora neste processo, dando voz e vez aos usuários, considerando seus conhecimentos, crenças, hábitos e papéis, de modo a assegurar a sustentabilidade da assistência em saúde ${ }^{(4,18)}$.

A assistência integral em saúde pressupõe trabalho multiprofissional, em que o foco é o bem-estar da criança. Nesta perspectiva, o enfermeiro, como membro desta equipe, deve ser atuante e valorizar a consulta de puericultura como estratégia para a promoção da saúde infantil e com vistas a produzir mudanças individuais e coletivas. A prática da consulta de enfermagem possibilita ao enfermeiro o resgate do cuidado, por meio de assistência sistematizada, estabelecendo corresponsabilidades com o usuário de forma a contribuir para a transformação do estilo de vida e da realidade sanitária no qual está inserido, suscitando a satisfação pessoal e valorização profissional do enfermeiro ${ }^{(19)}$.

No processo de cuidado da criança, o enfermeiro deve estar à frente das ações assistenciais e educativas, pois seu envolvimento é fundamental para o êxito do Programa de Puericultura na ESF. Porém, essas atividades devem ser compartilhadas pela equipe de saúde, de maneira que todos possam contribuir para o desenvolvimento saudável da criança. Neste contexto, os enfermeiros, assim como os outros profissionais, necessitam rever sua prática, buscando o aprimoramento e a qualidade no atendimento, além da resolutividade dos problemas cotidianos enfrentados na assistência infanti ${ }^{(10,20)}$.

\section{CONSIDERAÇÕES FINAIS}

A valorização e efetivação do PP pelos profissionais de saúde implicam na segurança e confiabilidade da comunidade no modelo preventivo de saúde. Desta forma, o paradigma médico hegemônico, com foco na medicalização e atendimento a demandas, perderá seu poder de influência no ideário predominante na população, dando vez a uma nova lógica de trabalho na ESF que vise à promoção da saúde da criança.

A consulta de puericultura realizada no âmbito da ESF constitui-se em instrumento indispensável na promoção da saúde infantil. Para tanto, necessita ser implementada em consonância com as diretrizes estabelecidas pelo Ministério da Saúde, adaptadas às necessidades de cada município.

Portanto, vale salientar a importância de se colocar o Programa de Puericultura, particularmente a consulta de puericultura, na pauta das discussões dos gestores municipais e responsáveis pelas equipes da ESF sobre o fazer cotidiano na atenção básica no contexto da promoção da saúde infantil. Há que se ressaltar, entre as diversas vantagens de incentivar-se esta prática, que se trata de um atendimento de baixa complexidade de execução e de baixo custo, em contraposição aos impactos positivos quando de sua implementação efetiva, no âmbito da qualidade de vida e saúde da população infantil. Considera-se ainda, ser de extrema importância o investimento em educação permanente dos profissionais atuantes e em processos avaliativos dos serviços de saúde, para que as práticas na atenção à saúde da criança estejam consonantes com as políticas públicas e diretrizes do SUS.

\section{REFERÊNCIAS}

1. Ministério da Saúde (BR). Secretaria de Atenção à Saúde. Área Técnica de Saúde da Criança e Aleitamento Materno. Gestões e gestores de políticas públicas de atenção à saúde da criança: 70 anos de história. Brasília (DF): MS;2011.

2. Ministério da Saúde (BR). Secretaria de Atenção à Saúde. Departamento de Ações Programáticas Estratégicas. Agenda de compromissos para a saúde integral da criança e redução da mortalidade infantil. Brasília (DF): MS;2004.

3. Erdmann A L, Sousa FGM. Cuidando da criança na Atenção Básica de Saúde: atitude dos profissionais de saúde. Mundo Saúde. 2009;33(2):150-60.

4. Vasconcelos VM, Frota MA, Martins MC, Machado MMT. Puericultura em enfermagem e educação em saúde: percepção de mães na estratégia saúde da família. Esc. Anna Nery 2012;16(2):326-31. 
5. Ministério da Saúde (BR). Secretaria de Atenção à Saúde. Departamento de Atenção Básica. Caderno de Atenção Básica. saúde da criança: crescimento e desenvolvimento. 33ed. Brasília (DF): MS; 2012.

6. Santos RKC, Resegue R, Puccini RF. Puericultura e a atenção à saúde da criança: aspectos históricos e desafios. Rev. bras. crescimento desenvolv. hum. [Internet] 2012;22(2) [acesso em 2013 jul 27]. Disponível: http://dx.doi.org/10.1590/S010412822012000200006

7. Portal do Departamento da Atenção Básica. Histórico da cobertura da Saúde da Família [Internet]. Brasília (DF); 2013 [atualizado 2013 set 30; acesso em 2013 out 28]. Disponível: http://dab.saude.gov.br/portaldab/ historico_cobertura_sf.php

8. Secretária de Saúde de Maringá. Plano Municipal de Saúde 2010/2013. [Internet] 2010 [acesso em 2013 out 28] Disponível: http://www2.maringa.pr.gov.br/ sistema/arquivos/edca39be41b9.pdf

9. Minayo MCS. Pesquisa social: teoria, método e criatividade. Petrópolis (RJ): Vozes; 2010.

10. Novaczyk AB, Dias NS, Gaíva MAM. Atenção à saúde da criança na rede básica: análise de dissertações e teses de enfermagem. Rev. Eletr. Enf. [Internet] 2008;10(4) [acesso em 27 jul 2013]. Disponível: http:// www.fen.ufg.br/revista/v10/n4/v10n4a25.htm

11. Del Ciampo LA, Ricco RG, Denaluzzi JC, Del Ciampo IRL, Ferraz IS, Almeida CAN. O programa de saúde da família e a puericultura. Ciênc. saúde coletiva. 2006;11(3):739-43.

12. Pinto JP, Ribeiro CA, Pettengill MM, Balieiro MMFG. Cuidado centrado na família e sua aplicação na enfermagem pediátrica. Rev. bras. enferm. 2010;63(1):132-5.

13. Monteiro Al, Macedo IP, Santos ADB, Araujo WM. A enfermagem e o fazer coletivo: acompanhando o crescimento e o desenvolvimento da criança. Rev Rene. 2011;12(1):73-80.

14. Assis WD, Collet N, Reichert APS, Sá LD. Processo de trabalho da enfermeira que atua em puericultura nas unidades de saúde da família. Rev Bras. 2011;64(1):38-46.

15. Neto FRGX, Queiroz CA, Rocha J, Cunha ICKO. Porque eu não levo meu filho para a consulta de puericultura. Rev. Soc. Bras. Enferm. Ped. 2010;10(2):51-9.

16. Gauterio DP, Irala DA, Cezar-Vaz MR. Puericultura em enfermagem: perfil e principais problemas encontrados em crianças menores de um ano. Rev. bras. enferm. 2012;65(3):508-13.

17. Martins PC, Cotta RMM, Mendes FF, Priore SE, Franceschini SCC, Cazal MM, et al. De quem é o
SUS? Sobre as representações sociais dos usuários do Programa Saúde da Família. Cien Saude Colet. 2011;16(3):1933-42.

18. Roecker S, Marcon SS. Educação em saúde. Relatos das vivências de enfermeiros com a Estratégia da Saúde Família. Invest. educ. enferm. 2011;29(3):381-90.

19. Campos RMC, Riberio CA, Silva CV, Saparolli ECL. Consulta de enfermagem em puericultura: a vivência do enfermeiro na Estratégia de Saúde da Família. Rev. esc. enferm. USP [Internet] 2011;45(3) [ acesso em 03 ago 2013]. Disponível: http://dx.doi.org/10.1590/ S0080-62342011000300003

20. Silveira VG, Martins MC, Albuquerque CM, Frota MA. Percepção da mãe sobre aleitamento materno na puericultura. Cienc Cuid Saude 2008;(4):523-29. 